\title{
Quantitative proteomic analysis reveals that serine/threonine kinase is involved in Streptococcus suis virulence and adaption to stress conditions
}

\author{
Haodan Zhu ${ }^{1,2,3}$. Junming Zhou ${ }^{1,2,3} \cdot$ Dandan Wang ${ }^{1,2,3} \cdot$ Zhengyu $\mathrm{Yu}^{1,2,3} \cdot \mathrm{Bin}^{\mathrm{Li}} \mathrm{i}^{1,2,3} \cdot$ Yanxiu $\mathrm{Ni}^{1,2,3}$. \\ Kongwang $\mathrm{He}^{1,2,3}$
}

Received: 4 December 2020 / Revised: 6 May 2021 / Accepted: 8 May 2021 / Published online: 24 May 2021

(c) The Author(s), under exclusive licence to Springer-Verlag GmbH Germany, part of Springer Nature 2021

\begin{abstract}
The eukaryotic-type serine/threonine kinase of Streptococcus suis serotype 2 (SS2) performs critical roles in bacterial pathogenesis. In this study, isobaric tags for relative and absolute quantification (iTRAQ) MS/MS were used to analyze the protein profiles of wild type strain SS2-1 and its isogenic STK deletion mutant ( $\Delta$ stk). A total of 281 significant differential proteins, including 147 up-regulated and 134 down-regulated proteins, were found in $\Delta$ stk. Moreover, 69 virulence factors (VFs) among these 281 proteins were predicted by the Virulence Factor Database (VFDB), including 38 downregulated and 31 up-regulated proteins in $\Delta$ stk, among which 15 down regulated VFs were known VFs of SS2. Among the down-regulated proteins, high temperature requirement $\mathrm{A}(\mathrm{HtrA})$, glutamine synthase $(\mathrm{Gln} A)$, ferrichrome $\mathrm{ABC}$ transporter substrate-binding protein FepB, and Zinc-binding protein AdcA are known to be involved in bacterial survival and/or nutrient and energy acquisition under adverse host conditions. Overall, our results indicate that STK regulates the expression of proteins involved in virulence of SS2 and its adaption to stress environments.
\end{abstract}

Keywords Streptococcus suis $\cdot$ Serine/threonine kinase $\cdot$ Quantitative proteomic $\cdot$ Virulence $\cdot$ Stress

\section{Introduction}

Streptococcus suis (S. suis) is a major swine pathogen that causes a wide range of diseases (Lun et al. 2007). In addition, $S$. suis is an important zoonotic agent responsible for severe human infections, including meningitis, endocarditis,

Communicated by Erko Stackebrandt.

Yanxiu Ni

er1998@126.com

Kongwang $\mathrm{He}$

kwh2003@263.net

1 Institute of Veterinary Medicine, Jiangsu Academy of Agricultural Sciences, Nanjing, People's Republic of China

2 Jiangsu Co-Innovation Center for the Prevention and Control of Important Animal Infectious Disease and Zoonose, Yangzhou University, Yangzhou, People's Republic of China

3 Key Lab of Food Quality and Safety of Jiangsu Province, State Key Laboratory Breeding Base, Nanjing, People's Republic of China and septic shock (Tang et al. 2006; Lun et al. 2007). Streptococcus suis serotype 2 (SS2), which is the serotype that is most virulent and most frequently isolated from diseased pigs, is most commonly involved in human infections (Tang et al. 2006). During the past few decades, more than 100 virulence factors of $S$. suis had been identified. These factors were classified into the following subgroups: surface/ secreted elements, enzymes/proteases, transcription factors/ regulatory systems and others (Fittipaldi et al. 2012). However, the mechanisms underlying the pathogenesis of the different virulence factors on SS2 has still not been entirely explained.

Bacteria use regulatory systems to sense and respond to environmental signals via regulation of specific gene expression. Two-component systems (TCSs), stand-alone regulators (SARs), regulator RNAs and unknown regulators constitute bacterial regulatory networks (Fittipaldi et al. 2012; Wu et al. 2014; Segura et al. 2017; Zheng et al. 2018a). Two-component systems such as SalK/SalR, VirR/VirS and VraSR, as well as the orphan response regulators RevS and CovR have been reported to contribute to bacterial adaptation to various environments and the expression of virulence 
factors of S. suis (Zheng et al. 2018a). In addition, several SARs have been reported to modulate $S$. suis virulence, including Rgg (Zheng et al. 2011), CcpA (Willenborg et al. 2011), CodY (Feng et al. 2016) and Rex (Zhu et al. 2018). Small RNAs also play a pivotal role in the pathogenicity of S. suis (Xiao et al. 2017).

Several recent studies have shown that eukaryote-type serine/threonine kinases (ESTKs) and phosphatases (ESTPs) also play essential roles in sensing of external stimuli (Wright and Ulijasz 2014). Even though signaling systems composed of ESTKs/ESTPs do not have dedicated transcription factors, they are capable of affecting the expression of genes involved in cell growth and division, adherence to host cell, stress response, biofilm formation, and various metabolic, developmental and virulence processes (Burnside and Rajagopal 2011; Zhu et al. 2011, 2014; Wright and Ulijasz 2014; Manuse et al. 2016; Fang et al. 2017; Zhang et al. 2017).

The homologues of ESTK and ESTP in S. suis, which have been designated SsSTK and SsSTP, respectively, were found to contribute to bacterial adherence to host cells, survival in stress environments and virulence.(Zhu et al. 2011, 2014; Fang et al. 2017; Zhang et al. 2017). Comparative proteome analysis is a powerful method for elucidating gene expression patterns in microorganisms (Chen et al. 2011; Pian et al. 2015; Yu et al. 2018). Proteome and transcriptome profiles are not exactly the same because of differences in the post-transcriptional regulation that modulates the translation rate and half-lives of specific proteins or mRNAs, as well as their intracellular location and molecular association with other proteins (Chen et al. 2011; Shen et al. 2013). A previous comparative transcriptomic analysis have shown that SsSTK affects the transcription of a set of genes encoding functions involving in cell growth and division, glycolysis, carbohydrate metabolism, membrane transport and translation (Zhang et al. 2017). In the present study, the isobaric tag for relative and absolute quantitation (iTRAQ) and liquid chromatography tandem mass spectrometry (LC-MS/MS) were used to analyze the different protein expression profiles, especially those concerning the bacterial pathogencity, between the wild-type (WT) strain SS2-1 and its SsSTK mutant strain with the aim of revealing proteins involved in adaption to stress environments and virulence of $S$. suis.

\section{Materials and methods}

\section{Bacterial strains and culture conditions}

SS2 strain SS2-1, which was isolated from a diseased pig with septicemia in Jiangsu Province in 1998, has been confirmed as a highly virulent strain (Zhu et al. 2014). The SsSTK mutant of SS2-1 $(\Delta s t k)$ was constructed in a previous study (Zhu et al. 2014). For this study, SS2 strains were grown in Todd-Hewitt broth (THB, Becton, Dickinson and Company, USA) or plated on agar medium containing $10 \%$ fetal bovine serum at $37^{\circ} \mathrm{C}$.

\section{Protein digestion and iTRAQ labelin}

Protein samples were performed with a few modifications as described by previous descriptions (Shen et al. 2013; Yu et al. 2018). Briefly, the WT strain SS2-1 and the mutant strain $\Delta s t k$ were each cultured in THB in triplicate (three SS2-1 and three $\Delta s t k)$ and collected during the log-growth phase $(\mathrm{OD} 600=0.7)$ (Shen et al. 2013; Yu et al. 2018). Cells were then centrifuged at $10,000 \times g$ for 5 min at $4{ }^{\circ} \mathrm{C}$, after which the pellets were washed twice with PBS (Shen et al. 2013). Comparative proteome analysis was subsequently performed at Wuhan GeneCreate Biological Engineering Co., Ltd. (Wuhan, GeneCreate, China). Protein digestion was performed as previously described (Jing et al. 2008), with some modifications. Briefly, SS2 cell pellets were ground to powder in liquid nitrogen and then incubated in dissolution buffer $(8 \mathrm{M}$ urea/100 $\mathrm{mM}$ triethylammonium hydrogen carbonate buffer (TEAB), pH 8.0) containing $1 \mathrm{mM}$ PMSF and 2 mM EDTA (final concentration) for $5 \mathrm{~min}$, after which $10 \mathrm{mM}$ DTT (final concentration) was added to the sample. Next, the suspension was sonicated for $15 \mathrm{~min}$ and then centrifuged at $4{ }^{\circ} \mathrm{C}$ at $14,000 \times g$ for $20 \mathrm{~min}$. The supernatant was subsequently mixed with four volumes of precooled acetone at $-20^{\circ} \mathrm{C}$ overnight. After another centrifugation, the resulting protein pellets were air-dried and resuspended in $8 \mathrm{M}$ urea/100 $\mathrm{mM}$ TEAB (pH 8.0). Protein samples were then reduced with $10 \mathrm{mM}$ DTT at $56{ }^{\circ} \mathrm{C}$ for $30 \mathrm{~min}$ and alkylated with $50 \mathrm{mM}$ iodoacetamide (IAM) for $30 \mathrm{~min}$ in the dark. Next, the protein concentration was measured using a Bradford Protein Assay Kit (Beyotime, Shanghai, China). After being diluted $5 \times$ with $100 \mathrm{mM}$ TEAB, equal amounts of proteins from each sample were used for tryptic digestion. Trypsin was added at an enzyme protein ratio of 1:50 (w/w), after which samples were digested at $37{ }^{\circ} \mathrm{C}$ for $12-16 \mathrm{~h}$. Following digestion, peptides were desalted using $\mathrm{C} 18$ columns and the resulting desalted peptides were dried under vacuum. The dried peptide powder was later re-dissolved with $0.5 \mathrm{M}$ TEAB and processed according to the manufacturer's instructions for the iTRAQ Reagent-8 plex Multiplex Kit (AB Sciex U.K. Limited). Three biological replicates of SS2-1 (1A, 1B, and 1C) were labeled with iTRAQ tags 113, 114, and 115, respectively, and three biological replicates of $\Delta s t k$ (20A, 20B, and 20C) were labeled with tags 116,117 , and 118 , respectively. The peptide samples were then fractionated using a Durashell C18 column $(5 \mu \mathrm{m}, 100 \AA$, $4.6 \times 250 \mathrm{~mm})$ on an Ultimate 3000 HPLC system (Thermo DINOEX, USA) operating at $1 \mathrm{ml} / \mathrm{min}$. Peptides were separated by increasing acetonitrile (ACN) concentrations under high $\mathrm{pH}(\mathrm{pH} 10)$ conditions and fractions were collected at $1 \mathrm{ml}$ intervals and pooled into 12 fractions. Each fraction was then dried under vacuum. 


\section{LC-ESI-MS/MS analysis}

Peptide samples were dissolved in $2 \%$ acetonitrile/ $0.1 \%$ formic acid and then analyzed using a Triple TOF $5600^{+}$mass spectrometer coupled with the Eksigent nanoLC System (SCIEX, USA) as previously described (Lin et al. 2015). The raw files collected from the Triple TOF 5600 were interpreted using ProteinPilot version 4.5 (July 2012, Applied Biosystems; Foster City, CA, USA). MS/MS spectra were searched against the Uniprot $S$. suis database (80,299 items, updated Jan 2017). For analysis, the instrument was set as TripleTOF 5600 plus with cysteine carbamidomethylation and 8 multiplex iTRAQ labeling was set as a fixed modification. In addition, methionine oxidation was used as a variable modification, and digestion by trypsin allowing for no more than one missed cleavage. The ratio of $\Delta s t k$ to SS2-1 represents the expression of proteins with a $1 \%$ false discovery rate for the protein identification confidence (Unwin et al. 2010). The differences in abundance were considered significant when whose criteria were met a ratio-fold change $\geq 1.5$ or $\leq 0.67$ (Yu et al. 2018).

\section{Bioinformatics analysis}

Gene Ontology (GO) analysis was conducted to classify differently expressed proteins (DEPs) in three categories (cell component, molecular function, and biological process) using the UniPort-GOA database (http://www.ebi.ac. uk/GOA/), InterProScan (http:// www.ebi.ac.uk/interpro/) and GO annotation (http://geneontology.org/). In addition, the Kyoto Encyclopedia of Genes and Genomes (KEGG) pathway (http://www.genome.jp/kegg/) of DEPs were further categorized utilizing the same resource. Furthermore, the protein-protein interactions for these DEPs predicted by the Virulence Factor Database (VFDB) (Chen et al. 2005) in this study and the known VFs reported by others were analyzed using the Cytoscape software to construct a virulence network of $S$. suis and screen the novel DEPs for those that are connected with the known VFs. The protein-protein interaction network was obtained from the STRING database (http://string-db.org/newstring_cgi/show_input_ page.pl), which defined a 'confidence score' to evaluate the interaction confidence. We obtained all interactions with a confidence score of at least 0.4 (Yu et al. 2018).

\section{Western blot validation of comparative proteomic analysis}

SS2-1 and $\Delta$ stk were prepared for western blot analysis. Two proteins, OppA (putative oligopeptide-binding protein) and DnaJ (chaperone protein DnaJ), were chosen for validation of the comparative proteomic data. In the $\Delta \mathrm{stk} / \mathrm{SS} 2-1 \mathrm{com}$ parison, OppA is a lower abundance protein and DnaJ is a higher abundance protein. EF-Tu was used as the loading control because its abundance is relatively constant. Equal amounts (30 $\mu \mathrm{g}$ for each lane) of whole cell proteins from the WT and mutant strains were separated on a $12 \%$ SDSPAGE gel, then transferred onto polyvinylidene fluoride (PVDF) membranes (BioRad). The membranes were then incubated with a 1:500 dilution of the primary antibodies for OppA and EF-Tu (kindly provided by Prof. Wei Zhang, Nanjing Agricultural University) and DnaJ (kindly provided by Prof. Weihuan Fang, Zhejiang University), after which they were incubated with horseradish peroxidase (HRP)-conjugated secondary antibody at a 1:10,000 dilution. Signals were detected using enhanced chemiluminescence (ECL) substrate (ThermoFisher Scientific).

\section{Statistical analysis}

The means of groups were compared using the Student's $t$ test (unpaired, two-tailed) in GraphPad Prism 5 (San Diego, USA), with a $P<0.05$ considered to be statistically significant.

\section{Results}

\section{Comparative proteome analysis of SsSTK mutant strain and WT strain}

iTRAQ coupled mass spectrometry identified a total of 1120 proteins from WT strain SS2-1 and its mutant strain $\Delta$ stk. Among these, the expression levels of 281 proteins differed significantly ( $>1.5$-fold change or $<0.67$-fold change, $P$ value $<0.05$ ) in $\Delta$ stk compared with its WT strain SS2-1, with $134(47.7 \%)$ down-regulated and 147 (52.3\%) up-regulated (Table S1).

\section{Functional classification annotation analysis of DEPs}

To gain insight into the functional categories of the 281 differentially expressed proteins (DEPs), GO analysis was performed to generate classification clusters based on biological process and molecular function. In the biological processes classification cluster, the five most enriched GO terms were biosynthetic processes (41 proteins [32.03\%] upregulated; 54 proteins [46.96\%] downregulated), nitrogen compound metabolic processes (49 proteins [38.28\%] upregulated; 30 proteins [26.09\%] downregulated), protein metabolic processes (26 proteins [20.31\%] upregulated; 39 proteins [33.91\%] downregulated), nucleotide and nucleic acid metabolic processes (41 proteins [32.03\%] upregulated; 14 proteins [28.1\%] downregulated), and carbohydrate metabolic processes (23 proteins [17.97\%] upregulated; 21 proteins [18.26\%] downregulated) (Fig. 1a). In the molecular 
Fig. 1 GO enrichment analysis of differently expressed proteins in $\Delta s t k$. Results are grouped by biological process (a) and molecular function (b)
A

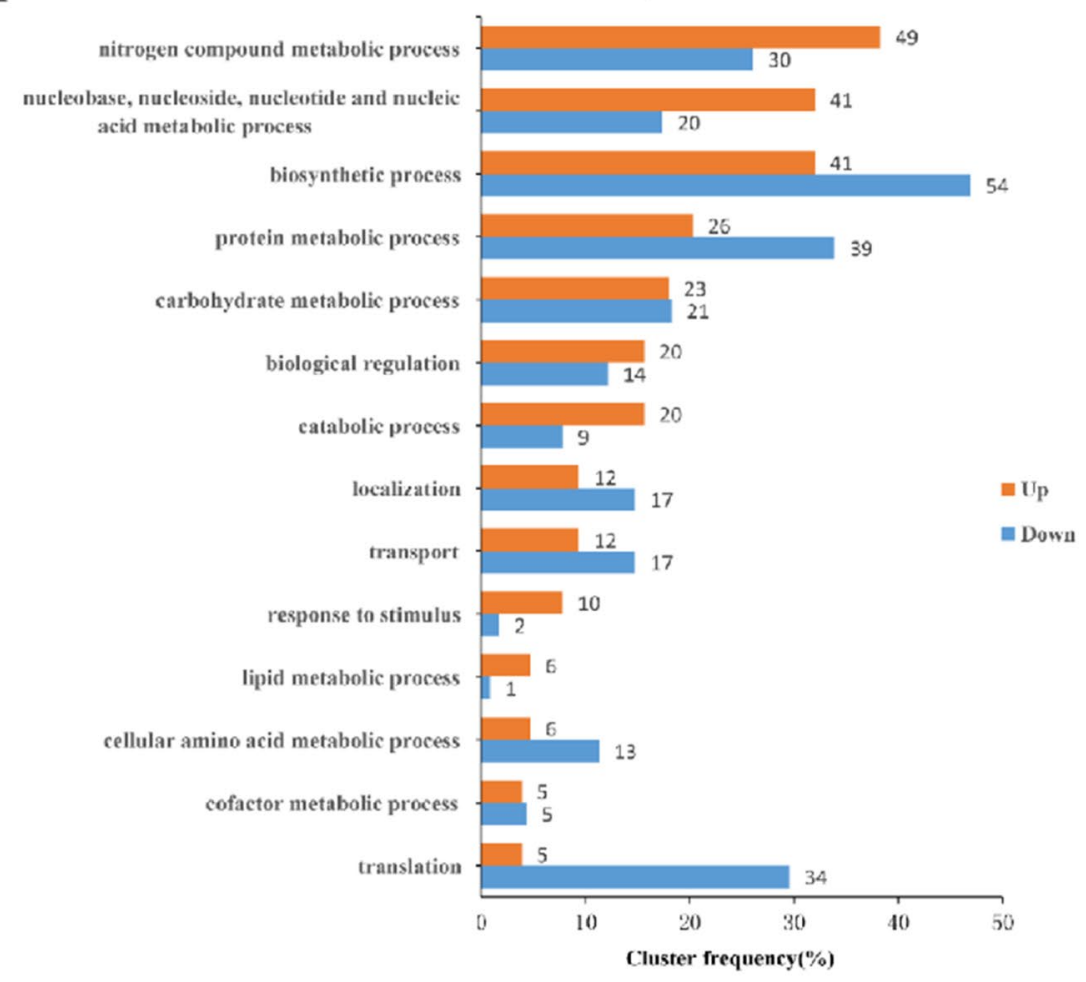

B

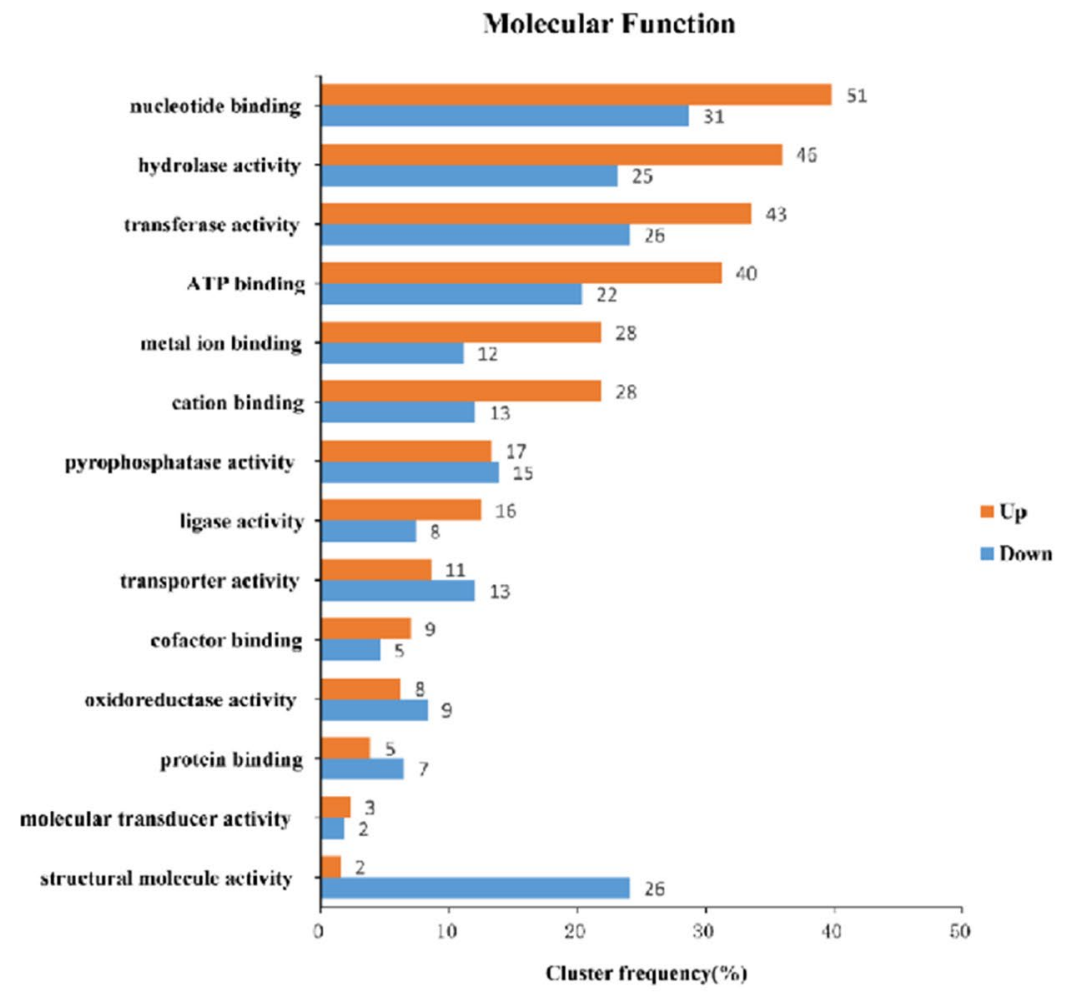

function classification, the most enriched GO terms were nucleotide binding (51 proteins [39.84\%] upregulated; 31 proteins [28.70\%] downregulated), hydrolase activity (46 proteins [35.94\%] upregulated; 25 proteins [23.15\%] downregulated), transferase activity (43 proteins [31.25\%] upregulated; 26 proteins [24.07\%] downregulated), ATP binding 
(46 proteins [39.84\%] upregulated; 25 proteins [20.37\%] downregulated), and cation binding (28 proteins [21.88\%] upregulated; 13 proteins [12.04\%] downregulated) (Fig. 1b).

\section{KEGG pathway analysis of DEPs}

To reveal the roles of SsSTK in SS2, KEGG pathway analysis was performed (Fig. 2). The DEPs were mainly involved in metabolic pathways (60 proteins [68.97\%] up-regulated; 43 proteins [55.13\%] down-regulated), biosynthesis of secondary metabolites (33 proteins [37.93\%] up-regulated; 21 proteins [26.92\%] down-regulated) and microbial metabolism in diverse environments (20 proteins [22.92\%] up-regulated; 11 proteins, [14.1\%] downregulated, Fig. 2a). The up-regulated proteins included those associated with purine metabolism (13 proteins, $14.94 \%$ ), $\mathrm{ABC}$ transporters (11 proteins, $12.64 \%$ ), fructose and mannose (10 proteins, $11.49 \%)$, propanoate (9 proteins, $10.34 \%$ ), peptidoglycan biosynthesis ( 8 proteins, $9.2 \%$ ), pyrimidine metabolism ( 8 proteins, $9.2 \%$ ) and pyruvate metabolism (7 proteins, $8.97 \%$ ). The downregulated pathways were associated with ribosomes $(26$ proteins, $33.3 \%$ ), $\mathrm{ABC}$ transporters (16 proteins, $20.51 \%$ ), purine metabolism (10 proteins, $12.82 \%$ ), pyrimidine metabolism (7 proteins, $8.97 \%$ ), amino sugar and nucleotide sugar metabolism (6 proteins, $7.69 \%$ ), the two-component system (5 proteins, $6.41 \%$ ) and aminoacyl-tRNA biosynthesis (5 proteins, 6.41\%) (Fig. 2b). In general, most of these DEPs are involved in key metabolic and pathways, which may contribute to the pathogenicity of SS2.

\section{SsSTK regulates known virulence factors}

The SsSTK deletion significantly reduced SS2 virulence. Among the 281 DEPs, there were 69 virulence factors (VFs) predicted by VFDB, including 38 down-regulated proteins (Table 1) and 31 up-regulated proteins (Table 2) in the $\Delta s t k$, of which 26 were known VFs of SS2 (Fittipaldi et al. 2012). In addition, the following $16 \mathrm{VFs}$ were down-regulated in $\Delta s t k$ : capsular polysaccharide biosynthesis locus genes CPS2A (regulation), CPS2B (chain length determination) and CPS2H (glycosyltransferase), sialic acid synthase (NeuB), UDP-N-acetylglucosamine 2-epimerase (NeuC), putative oligopeptide-binding protein (OppA), high-affinity zinc uptake system protein (ZnuA/ TroA) and IgM protease (IdeSsuis) (Fittipaldi et al. 2012; Rungelrath et al. 2018). Additionally, the putative IgAspecific zinc metalloproteinase (ZmpC or IgA1) (Zhang et al. 2011; Dumesnil et al. 2018), translation initiation factor 2 (HP0272 or SadP) (Ferrando et al. 2017), chaperone protein DnaJ (Zhang et al. 2015), and sensor histidine kinase TCS VarS (Zheng et al. 2018a; Zhong et al. 2018) were significantly up-regulated in the mutant strain. Another 43 VFs that have been reported in other pathogens were identified as DEPs in the $\Delta s t k$, including putative 5'-nucleotidase (5NuC) (Zheng et al. 2015; Ma et al. 2017), trypsin-like serine protease (HtrA) (Backert et al. 2018) and metalloendopeptidas (PepO) (Agarwal et al. 2013, 2014). These VFs were mainly assigned into eight classes: (i) amino acid transport and metabolism (12 proteins); (ii) posttranslational modification, protein turnover, chaperones (10 proteins); (iii) nucleotide transport and metabolism (9 proteins); (iv) transcription (8 proteins);
Fig. 2 KEGG pathway enrichments in DEPs in $\Delta$ stk. a Upregulated and $\mathbf{b}$ down-regulated proteins
A

Statistics of Up-Regulated Proteins

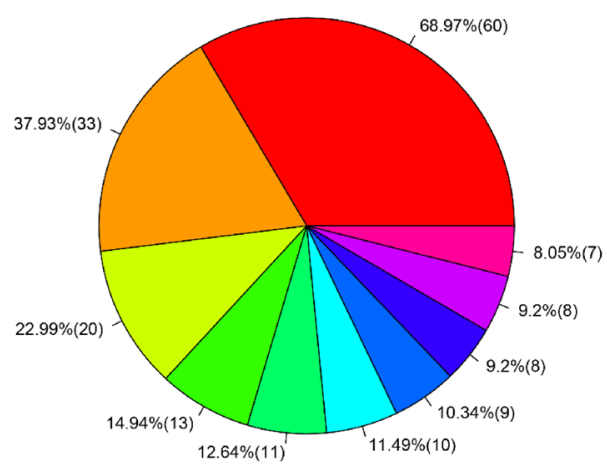

- Metabolic pathways

Biosynthesis of secondary metabolites

Microbial metabolism in diverse environments

Purine metabolism

ABC transporters
Fructose and mannose metabolism

Propanoate metabolism

- Peptidoglycan biosynthesis

- Pyrimidine metabolism
B Statistics of Down-Regulated Proteins

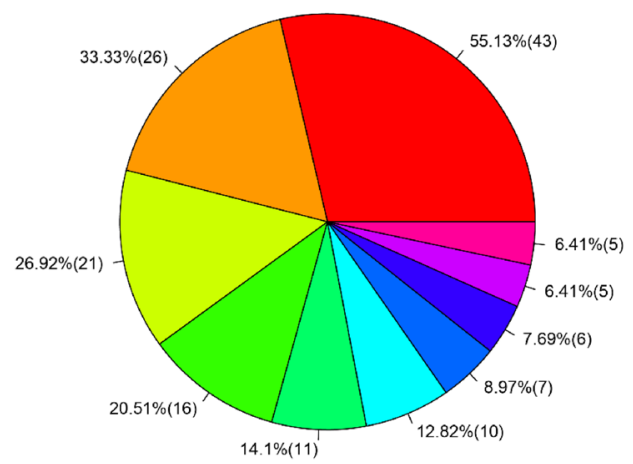

Metabolic pathways

- Ribosome

of secondary metabolites

ABC transporters

Microbial metabolism in diverse environments

- Pyrimidine metabolism

Amino sugar and nucleotide sugar metabolism

Two-component system

- Aminoacyl-tRNA biosynthesis 
Table 1 Down-regulated virulence associated factors identified by iTRAQ in $\Delta$ stk

\begin{tabular}{|c|c|c|c|c|c|}
\hline Accession & Gene locus & Gene name & Description/Function & $\begin{array}{l}\text { Fold change } \\
\text { Mutant/WT }\end{array}$ & $P$ value \\
\hline \multicolumn{6}{|c|}{ Amino acid transport and metabolism } \\
\hline A0A116MPN5 & ssu05-1868 & OppA & Putative oligopeptide-binding protein OppA & 0.260903997 & 0 \\
\hline G7SK61 & ssu05-1027 & $\mathrm{GlnP}$ & $\begin{array}{l}\text { Putative glutamine } \mathrm{ABC} \text { transporter, glutamine-binding protein/per- } \\
\text { mease protein }\end{array}$ & 0.412407484 & $2.96 \mathrm{E}-05$ \\
\hline A4VSY1 & ssu05_0252 & GDH & Glutamate dehydrogenase & 0.360913896 & 4.91E-08 \\
\hline A4VSP1 & ssu05-0160 & $\mathrm{Gln} \mathrm{A}$ & Glutamine synthetase & 0.318288584 & $1.10 \mathrm{E}-05$ \\
\hline A4VXL3 & ssu05_1886 & $\mathrm{IlvC}$ & Ketol-acid reductoisomerase & 0.598600521 & $6.38 \mathrm{E}-05$ \\
\hline A4VY44 & ssu05_2067 & GlnQ & Putative amino acid $\mathrm{ABC}$ transporter & 0.651608966 & 0.0284189 \\
\hline A0A0K2E4M4 & ssu05-1548 & LivK & $\begin{array}{l}\text { Branched-chain amino acid ABC transporter substrate-binding pro- } \\
\text { tein LivK }\end{array}$ & 0.626362412 & 0.00362246 \\
\hline A0A0Z8K531 & ssu05_0718 & ASD & Aspartate-semialdehyde dehydrogenase & 0.597290304 & 0.00686381 \\
\hline \multicolumn{6}{|c|}{ Nucleotide transport and metabolism } \\
\hline A0A0H3MXU4 & ssu05_2118 & NrdD & Anaerobic ribonucleoside-triphosphate reductase & 0.262453737 & $5.79 \mathrm{E}-10$ \\
\hline A0A0Z8I535 & ssu05_0873 & GuaA & GMP synthase & 0.572778731 & 0.00943322 \\
\hline A4VSH4 & ssu05_0091 & $\mathrm{ADK}$ & Adenylate kinase & 0.433358178 & $9.74 \mathrm{E}-05$ \\
\hline A4VWL5 & ssu05-1538 & $5 \mathrm{NuC}$ & Putative 5'-nucleotidase & 0.34182614 & $1.38 \mathrm{E}-06$ \\
\hline A4VU67 & ssu05_0690 & Fhs & Formate-tetrahydrofolate ligase & 0.538793986 & 0.00305715 \\
\hline A0A0Z8Y8J2 & ssu05-0033 & PurD & Phosphoribosylamine-glycine ligase purD & 0.441863004 & 0.03455731 \\
\hline \multicolumn{6}{|c|}{ Cell wall/membrane/envelope biogenesis } \\
\hline A4VTS6 & ssu05_0549 & GlmS & Glutamine-fructose-6-phosphate aminotransferase & 0.237614116 & 8.34E-08 \\
\hline A4VVX1 & ssu05_1294 & AltA & $\mathrm{N}$-acetylmuramoyl-L-alanine amidase & 0.500737006 & 0.00053377 \\
\hline A4VTV5 & ssu05_0578 & $\mathrm{NeuB}$ & Sialic acid synthase & 0.363283669 & 0.00013004 \\
\hline A4VTV6 & ssu05_0579 & $\mathrm{NeuC}$ & UDP-N-acetylglucosamine 2-epimerase & 0.561224277 & 0.04520204 \\
\hline A4VTU2 & ssu05_0565 & Cps2B & CPS biosynthesis ( chain length determination) & 0.519610055 & 0.0295358 \\
\hline \multicolumn{6}{|c|}{ Inorganic ion transport and metabolism } \\
\hline U5UKP6 & ssu05-1771 & MetQ & Lipoprotein & 0.467159782 & $3.22 \mathrm{E}-05$ \\
\hline A4VSJ3 & ssu05-0112 & AdcA & Zinc-binding protein AdcA & 0.312008388 & $2.30 \mathrm{E}-06$ \\
\hline A4VU25 & ssu05-0649 & FepB/FhuD & Ferrichrome $\mathrm{ABC}$ transporter substrate-binding protein & 0.371811659 & $7.41 \mathrm{E}-05$ \\
\hline A0A0M9FIU4 & ssu05-0309 & ZosA & Cation transport ATPase ZosA & 0.211173974 & 0.00041522 \\
\hline D5AIQ2 & ssu05-1418 & MgtA & Magnesium-transporting ATPase & 0.567922803 & 0.00017229 \\
\hline \multicolumn{6}{|l|}{ Transcription } \\
\hline A4VTU1 & ssu05_0564 & Cps2A & CPS biosynthesis (regulation) & 0.486937335 & 0.03900053 \\
\hline D5AFJ8 & ssu05-0159 & GlnR & Transcriptional regulator, glutamine synthetase repressor & 0.289088662 & 0.02195271 \\
\hline A4VV72 & ssu05_1045 & GlpR & Transcriptional regulator of sugar metabolism & 0.573794895 & 0.04839645 \\
\hline A0A123TNJ0 & ssu05-0402 & YebC & Probable transcriptional regulatory protein yebC & 0.322587925 & 0.00155311 \\
\hline \multicolumn{6}{|l|}{ Function unknown } \\
\hline A0A0H3MYM8 & ssu05_1267 & InlA & Streptococcal histidine triad-family protein & 0.585290914 & 0.00207815 \\
\hline A4VTU8 & ssu05_0571 & $\mathrm{Cps} 2 \mathrm{H}$ & CPS biosynthesis (glycosyltransferase) & 0.59434387 & 0.00572005 \\
\hline A0A0H3MW10 & ssu05-545 & IdeSsuis & IgM protease & 0.389281421 & $2.94 \mathrm{E}-05$ \\
\hline B3GFZ7 & ssu05-0177 & $\mathrm{EF}$ & Extracellular factor protein & 0.298326271 & 0.00592074 \\
\hline A0A123TR75 & ssu05-0141 & Impdh & Inosine-5' -monophosphate dehydrogenase & 0.611950148 & 0.03139697 \\
\hline U5UEH8 & ssu05-1257 & PBP1 & $\mathrm{ABC}$ transporter permease protein & 0.202037287 & 0.00087703 \\
\hline \multicolumn{6}{|c|}{ Energy production and conversion } \\
\hline A0A0H3MXX6 & ssu05-2154 & FrdA & Putative fumarate reductase flavoprotein subunit & 0.228390668 & 0.00000546 \\
\hline \multicolumn{6}{|c|}{ Signal transduction mechanisms } \\
\hline A4VTF6 & ssu05-0428 & STK & Serine/threonine protein kinase & 0.042032747 & $1.71 \mathrm{E}-10$ \\
\hline \multicolumn{6}{|c|}{ Posttranslational modification, protein turnover, chaperones } \\
\hline A4VXJ8 & ssu05-1871 & SufB & FeS assembly protein SufB & 0.495251882 & 0.00073167 \\
\hline A4VYG9 & ssu05-2192 & HtrA & Trypsin-like serine protease HtrA & 0.611081572 & 0.0303447 \\
\hline
\end{tabular}


Table 2 Up-regulated virulence associated factors identified by iTRAQ in $\Delta$ stk

\begin{tabular}{|c|c|c|c|c|c|}
\hline Accession & Gene locus & Gene name & Description/function & Fold change mutant/WT & $P$ value \\
\hline \multicolumn{6}{|c|}{ Posttranslational modification, protein turnover, chaperones } \\
\hline A4VSA0 & ssu05-0015 & FtsH & ATP-dependent zinc metalloprotease FtsH & 2.666489219 & $8.88 \mathrm{E}-10$ \\
\hline A4VY59 & ssu05-2082 & PepgO & Predicted metalloendopeptidase & 1.592715723 & $5.40 \mathrm{E}-05$ \\
\hline A4VTN2 & ssu05_0505 & Collagenase & Collagenase and related protease & 2.58065221 & 0.00085438 \\
\hline A4VT31 & ssu05_0302 & DnaJ & Chaperone protein DnaJ & 5.099121717 & $1.38 \mathrm{E}-10$ \\
\hline A0A116NL00 & ssu05-1737 & $\operatorname{TrxB}$ & Thioredoxin reductase & 1.587025457 & 0.00379886 \\
\hline A4VTN3 & ssu05_0506 & HP0506 & Collagenase and related protease & 1.512746775 & 0.01979603 \\
\hline G7SNC3 & ssu05-0240 & MrsA & Peptide methionine sulfoxide reductase & 1.798334701 & 0.02091889 \\
\hline A4VT28 & ssu05_0298 & GrpE & Protein GrpE & 1.639950423 & 0.00096526 \\
\hline \multicolumn{6}{|c|}{ Amino acid transport and metabolism } \\
\hline A4VVS0 & ssu05_1243 & PepF & Oligoendopeptidase F & 2.335395064 & $6.57 \mathrm{E}-08$ \\
\hline A4VXS8 & ssu05-1951 & AmpT & Leucyl aminopeptidase & 1.68826166 & 0.00091845 \\
\hline A0A0Z8CXK2 & ssu05_0728 & PepF1-2 & Oligoendopeptidase F & 2.102440266 & 0.00069887 \\
\hline A4VU01 & ssu05_0624 & ArcA & Arginine deiminase & 2.592388155 & $1.04 \mathrm{E}-06$ \\
\hline \multicolumn{6}{|l|}{ Transcription } \\
\hline A4VUG1 & $\operatorname{ssu05-0784}$ & CpsY & CpsY & 1.665254688 & 0.00096483 \\
\hline A0A168YWM4 & ssu05-1012 & LysR & LysR family transcriptional regulator & 1.597409841 & 0.02397035 \\
\hline A0A0Z8IRK2 & ssu05_1700 & ScpB & Segregation and condensation protein B & 1.51173895 & 0.02841734 \\
\hline A4VT27 & ssu05_0299 & $\mathrm{HrcA}$ & Heat-inducible transcription repressor $\mathrm{HrcA}$ & 3.027403522 & 0.04096617 \\
\hline \multicolumn{6}{|c|}{ Nucleotide transport and metabolism } \\
\hline A4VVU8 & ssu05_1271 & $\mathrm{Cmk}$ & Cytidylate kinase & 1.650953089 & 0.02280986 \\
\hline A4VUL1 & ssu05_0834 & ThyA & Thymidylate synthase & 1.540254675 & 0.02311404 \\
\hline D5AK71 & ssu05_1966 & PurA & Adenylosuccinate synthetase & 1.677929485 & 0.00077763 \\
\hline \multicolumn{6}{|c|}{ Signal transduction mechanisms } \\
\hline A4VTF8 & ssu05_0430 & VarS & Sensor histidine kinase VarS & 1.645401188 & 0.00250477 \\
\hline A4VWT3 & ssu05_1606 & HprK & HPr kinase/phosphorylase & 1.53464737 & 0.00154572 \\
\hline \multicolumn{6}{|c|}{ General function prediction only } \\
\hline A4VSS6 & ssu05-0197 & PepX & Xaa-Pro dipeptidyl-peptidase & 1.836436817 & 0.00597575 \\
\hline D5AIW8 & ssu05-1495 & TPR & Tetratricopeptide repeat family protein & 1.560591535 & 0.0141768 \\
\hline \multicolumn{6}{|c|}{ Function unknown } \\
\hline A0A0H3MV01 & ssu05_1022 & $\mathrm{ZmpC}$ & Putative IgA-specific zinc metalloproteinase & 2.251180198 & $1.52 \mathrm{E}-13$ \\
\hline A4VSS5 & ssu05-0196 & Fhbp & Uncharacterized protein & 5.347251049 & $8.88 \mathrm{E}-16$ \\
\hline \multicolumn{6}{|c|}{ Inorganic ion transport and metabolism } \\
\hline A0A0Z8XZK6 & ssu05-0658 & TelA & Tellurite resistance protein & 1.581032077 & 0.00013611 \\
\hline \multicolumn{6}{|c|}{ Replication, recombination and repair } \\
\hline A4VVA2 & ssu05_1075 & GyrA & DNA gyrase subunit A & 1.663559748 & 0.00072441 \\
\hline \multicolumn{6}{|c|}{ Intracellular trafficking, secretion, and vesicular transport } \\
\hline A4VUQ3 & ssu05_0876 & Ffh & Signal recognition particle protein & 1.822934548 & 0.00080988 \\
\hline \multicolumn{6}{|c|}{ Carbohydrate transport and metabolism } \\
\hline A4VU63 & ssu05_0686 & PMM & Phosphomannomutase & 1.584042233 & 0.00014063 \\
\hline \multicolumn{6}{|c|}{ Lipid transport and metabolism } \\
\hline G7SMB7 & ssu05_1807 & $\mathrm{FabH}$ & 3-oxoacyl-[acyl-carrier-protein] synthase 3 & 1.534641502 & 0.00468633 \\
\hline \multicolumn{6}{|c|}{ Cell wall/membrane/envelope biogenesis } \\
\hline A4VT01 & ssu05-0272 & SadP/IF-2 & Translation initiation factor 2 (IF-2 GTPase) & 5.605539481 & $2.22 \mathrm{E}-16$ \\
\hline
\end{tabular}


(v) inorganic ion transport and metabolism (6 proteins); (vi) cell wall/membrane/envelope biogenesis (6 proteins); (vii) general function prediction (3 proteins); and (viii) other proteins of unknown function (7 proteins) (Tables 1 and 2).

\section{Interaction network analysis confirmed the roles of the newly identified DEPs in the known virulence factor system}

For further insight into the roles of these newly identified DEPs in virulence, we visualized the network formed by the known VFs of SS2 and the novel identified VFs predicted by VFDB using the Cytoscape software. The protein-protein interaction network demonstrated 241 direct physical interactions among the 80 nodes (Table S2). Sixty-six of the interactions had a score higher than 0.70 (high confidence). The 36 newly identified DEPs (yellow nodes) were implicated in and complemented the virulence interaction network, with some playing an important role of bridging to link the known VFs (green nodes and red nodes) and forming important hub proteins. Overall, the results indicated that 37 of the 69 novel DEPs are involved in the known virulence network and may play a role in virulence (Fig. 3).

\section{Confirmation of the proteomics results by western blot analysis}

The up-regulated VF DnaJ (41kD) and down-regulated VF OppA $(66 \mathrm{kD})$ were selected for confirmation of the comparative proteomics analysis. The EF-Tu protein was used as an internal reference because its abundance is relatively constant in both groups. The western blot results support those of the proteomic analysis, as the levels of OppA was decreased and the levels of DnaJ were increased in $\Delta s t k$, which indicating that the proteomics data and western blot results agreed (Fig. 4).

\section{Discussion}

Bacterial ESTKs have emerged as important regulation elements that are indispensable for pathogenesis (Burnside and Rajagopal 2011; Wright and Ulijasz 2014; Manuse et al. 2016). In Mycobacterium tuberculosis, two-dimensional gel electrophoresis was used to investigate the effects of the serine/threonine protein kinase (pknE) on the bacterial protein expression under nitric oxide stress conditions. In response to NO stress, $\Delta \mathrm{pknE}$ had increased number of proteins involved in intermediary and lipid metabolism (Parandhaman et al. 2014). In S.pneumoniae, a mass-spectrometry based label-free quantitative (LFQ) approach was used to characterize and determine the impact of StkP on the protein expression profiles. Notable changes in the proteome of the kinase mutant $\Delta$ stkP in comparison to the WT strain have been observed especially in the cluster of amino acid metabolism, energy metabolism, regulatory fuction and transcription(Hirschfeld et al. 2019). In the present study, comparative proteomics approaches revealed that SsSTK can regulate the expression of proteins involved in bacterial central metabolism, stress response and virulence. These findings provide further support for the previous data that showed attenuated growth in vivo, reduced survival rate in
Fig. 3 Interaction networks of differentially expressed proteins predicted by the VFDB created using Cytoscape. Protein-protein interactions of differentially expressed proteins of S.suis that had a confidence score $\geq 0.4$ were visualized. The lines represent the interactions that exist between proteins

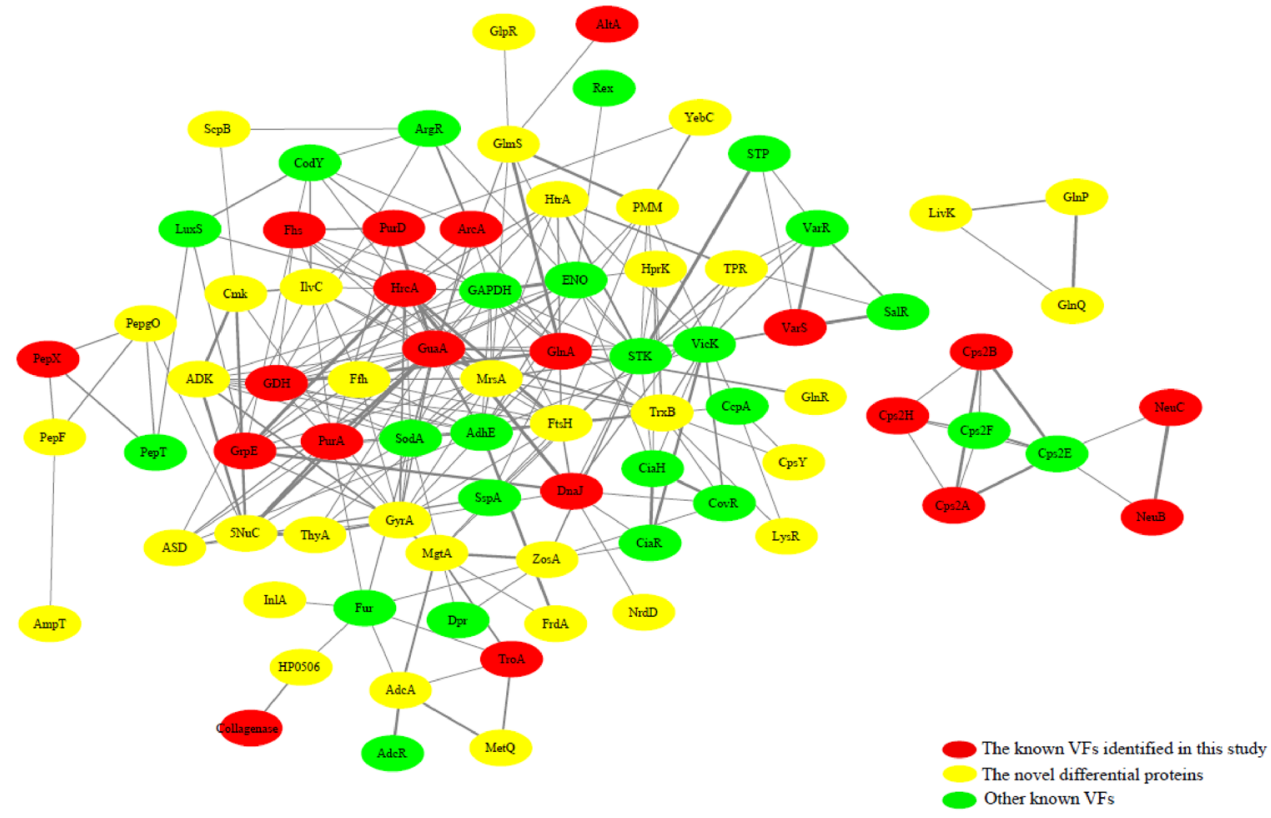




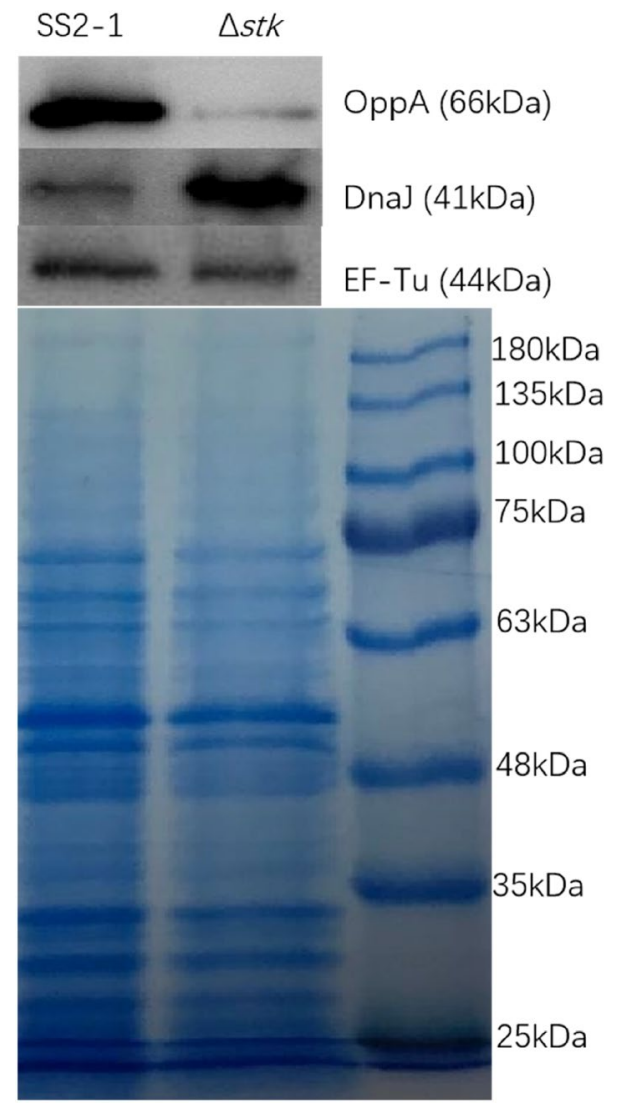

Fig. 4 Western blot analysis of comparative proteomics data. Equal amounts ( $30 \mu \mathrm{g}$ for each lane) of total bacterial cell proteins were separated on a $12 \%$ SDS-PAGE gel, then subjected to western blotting. From left to right the lanes were loaded with SS2-1 and $\Delta$ stk samples. Differentially expressed OppA (66 kDa, the first line), DnaJ (41 kDa, the second line) and EF-Tu (44 $\mathrm{kDa}$, the last line) proteins were analyzed using their respective antibodies. The CBB-R250-stained gel was used as a loading control. Protein bands were visualized using the ECL substrate

various stress environments and virulence of $\Delta s t k$ (Zhu et al. 2014).

As previously reported, the deletion of stk in strain SS2 resulted in alteration of bacterial pathogenicity (Zhu et al. 2014; Zhang et al. 2017). This attenuation may result from the impaired growth of $\Delta s t k$ in vivo and because of direct effects on the expression of VFs. The transcriptomic profiles showed that 32 VFs were down-regulated in stkdeletion strain of SC-19, including 9 known VFs of SS2, such as subtilisin-like protease (SspA), DNA nuclease (SsnA), mannose-specific PTS (ManN), adenylosuccinate synthase (PurA) and phosphoribosylamine-glycine ligase (PurD) (Wilson et al. 2007; Fittipaldi et al. 2012; Zhang et al. 2017). Our iTRAQ analysis showed 38 VFs, including 26 known VFs and 12 novel identified VFs that were predicted by VFDB, with repressed expression in $\Delta s t k$ such as AtlA, IdeSsuis, OppA, HtrA, HtpsB, PurD and 5NuC
(Fittipaldi et al. 2012). In SS2 virulent strain HA9801, the AtlA mutant strain $(\Delta a t l A)$ exhibited a significant reduction in adherence to epithelial cells, biofilm formation and virulence (Ju et al. 2012). In SS2 virulent strain 10, IgM cleaving activity of IdeSsuis is important for bacterial survival in porcine blood and evasion of the classical complement pathway (Rungelrath et al. 2018). In Strptococci, HtrA involved in tissue invasion, chronic airway infections and secretion of VFs, such as in S. pyogenes (Lyon and Caparon 2004) and S. pneumonia (Ibrahim et al. 2004). The histidine triadfamily protein Htps B, a homologue of internalin A (InlA) of Listeria monocytogenes, has been reported to be important in surface invasions of bacteria and to facilitate both bacterial attachment and internalization in cells that express its receptor of E-cadherin (Bergmann et al. 2002). In SS2 virulent strains, OppA is involved in cell growth, binding to host proteins and virulence (Zhang et al. 2014; Zheng et al. 2018b). In SS2 strain S735, purD insertion mutant strain obtained by the signature-tagged mutagenesis system was showed apathogenic in both mouse and caesarian-derived, colostrum-deprived (CDCD) pig models (Wilson et al. 2007). Recently transcriptome profiles and our proteome analysis both showed that PurD were down-regulated in stk deletion mutant strain (Zhang et al. 2017). The extracellular nucleases and or nucleotidase play important roles in degradation of the DNA backbone of neutrophil extracellular traps (NETs) and allow bacteria to evade the host immune system. In S. equi subsp. zooepidemicus ATCC35246, 5' -Nucleotidase $(5 \mathrm{NuC})$ was found to directly degrade the NET DNA backbone to deoxyadenosine, negatively influencing macrophage phagocytic activity, while the mutant strain $\Delta 5$ nuc exhibited lower virulence and a weaker ability to spread from blood to organs than the WT strain (Ma et al. 2017). Similar findings were reported in S. pyogenes for 5'-nucleotidase A (S5nA)(Zheng et al. 2015). Therefore, our results are in agreement those of previous studies showing SsSTK mutant strains displayed reduced ability to adhere to epithelial cells, increased immune evasion and increased sensitivity to phagocytosis (Zhu et al. 2014; Zhang et al. 2017).

During infection, bacteria must often cope with stress in the form of oxidative conditions, excessive temperature, extreme osmolarity, low $\mathrm{pH}$, and nutrient limitations. Our previous study showed that $\Delta s t k$ displayed defects in the ability to adapt to various environmental conditions ( $\mathrm{Zhu}$ et al. 2014). These may be because of down-regulation of the following series of DEPs that are necessary for bacterial survival and/or nutrient and energy acquisition under adverse host conditions. (i) The general stress response protein (e.g., HtrA). The virulence features of bacterial HtrA, an important stress response regulation protein, have primarily been attributed to increased fitness of pathogens because of resistance against stress conditions during infection (Backert et al. 2018). (ii) Amino acid transport and 
metabolism (e.g., GlnA, IlvC, LivK and ASD). Glutamine synthase $(\mathrm{G} \ln \mathrm{A})$, which converts glutamate and ammonia to glutamine, plays a central role in regulating the carbon/ nitrogen balance in the metabolism and the pathogenicity of bacteria, such as in Salmonella enterica and SS2 (Si et al. 2009; Aurass et al. 2018). The ketol-acid reductoisomerase IlvC and branched-chain amino acid ABC transporter substrate-binding protein LivK are involved in the branchedchain amino acids (BCAAs; isoleucine, leucine, valine) biosynthesis pathway, which contributes to the virulence of pathogens (Ribardo and Hendrixson 2011; Kim et al. 2017). In S. pneumoniae strain D39, an ilvC deletion mutant $(\Delta i l v C)$ diminished Ply and LytA virulence factor expression and showed a higher survival rate and lower bacterial burden in a mice infection model relative to the WT strain (Kim et al. 2017). Aspartate-semialdehyde dehydrogenase (ASD) is an essential enzyme for the biosynthesis of lysine, methionine, and threonine from aspartate. In Burkholderia pseudomallei, a deletion mutant strain $\Delta$ asd exhibited attenuated intracellular infectivity and the mutation showed protection against acute inhalation melioidosis in mice (Norris et al. 2011). (iii) Inorganic ion transport and metabolism (e.g., AdcA, FepB and MgtA). Apart from the two DEPs mentioned above, these proteins can help pathogens overcome the hostile environments created by ion starvation. The Adc protein contributes zinc uptake and streptococcal virulence. In $S$. agalactiae, Adc and Lmb are involved in zinc acquisition and contribute to bacterial growth and survival (Moulin et al. 2016). In S. pneumoniae, AdcA enables zinc acquisition during growth in vitro and systemic virulence in vivo (Bayle et al. 2011; Plumptre et al. 2014). In Salmonella enterica serovar Typhimurium wild-type strain SL1344, deletion of $f e p B$ attenuated Salmonella replication and colonization within macrophages and mice (Nagy et al. 2013). Moreover, the transcriptional level of $m g t A$ in $\Delta s t k$ was found to be decreased in previous studies (Zhang et al. 2017). Down-regulation of these VFs may hinder the acquisition of nutrients by bacteria and therefore decrease the adaptation of SS2 to various stress environments.

\section{Conclusion}

In summary, our comparative proteome analysis identified 38 down-regulated VFs in the mutant strain $\Delta s t k$ that were involved in adherence to host cells and adaption to and survival in the host environments during SS2 infection. Consistently, phenotypic assays in previous studies have confirmed that the $\Delta s t k$ mutant strain displayed deficient growth in various stress environments in vitro and in vivo and attenuated pathogenicity. Therefore, STK is important to cell growth, stress response, and virulence of SS2.
Supplementary Information The online version contains supplementary material available at https://doi.org/10.1007/s00203-021-02369-5.

Acknowledgements We thank Liwen Bianji, Edanz Group China (www.liwenbianji.cn/ac), for editing the English text of a draft of this manuscript.

Author contributions Conceived and designed the experiments: HZ, YN and KH. Performed the experiments: HZ, JZ and DW. Analyzed the data: $\mathrm{HZ}$ and JZ. Contributed reagents/materials/analysis tools: ZY, BL, YN, and KH. Wrote the manuscript: HZ, YN and KH. All authors read, advised, and approved the final manuscript.

Funding This work was supported by National Key Research and Development Program (2018YFD0500101), National Natural Sciences Foundation of China (31302114), the Special Fund for Public Welfare Industry of Chinese Ministry of Agriculture (201303041), and the Innovation of Agricultural Sciences in Jiangsu province (CX(14)5042). The funders had no role in the study design, data collection and analysis, decision to publish, or preparation of the manuscript. Comparative proteomics were performed with the help of Wuhan GeneCreate Biological Engineering Co., Ltd.

Data availability All data during the study appear in the submitted article and the supplementary materials.

\section{Declarations}

Conflict of interest The authors declare no conflict of interest.

\section{References}

Agarwal V, Kuchipudi A, Fulde M, Riesbeck K, Bergmann S, Blom AM (2013) Streptococcus pneumoniae endopeptidase O (PepO) is a multifunctional plasminogen- and fibronectin-binding protein, facilitating evasion of innate immunity and invasion of host cells. J Biol Chem 288:6849-6863. https://doi.org/10.1074/jbc.M112. 405530

Agarwal V, Sroka M, Fulde M, Bergmann S, Riesbeck K, Blom AM (2014) Binding of streptococcus pneumoniae endopeptidase $\mathrm{O}$ (PepO) to complement component $\mathrm{C} 1 \mathrm{q}$ modulates the complement attack and promotes host cell adherence. J Biol Chem 289:15833-15844. https://doi.org/10.1074/jbc.M113.530212

Aurass P, Duvel J, Karste S, Nubel U, Rabsch W, Flieger A (2018) glnA truncation in salmonella enterica results in a small colony variant phenotype, attenuated host cell entry, and reduced expression of flagellin and SPI-1-associated effector genes. Appl Environ Microbiol. https://doi.org/10.1128/AEM.01838-17

Backert S, Bernegger S, Skorko-Glonek J, Wessler S (2018) Extracellular HtrA serine proteases: an emerging new strategy in bacterial pathogenesis. Cell Microbiol 20:e12845. https://doi.org/10.1111/ cmi. 12845

Bayle L, Chimalapati S, Schoehn G, Brown J, Vernet T, Durmort C (2011) Zinc uptake by streptococcus pneumoniae depends on both AdcA and AdcAII and is essential for normal bacterial morphology and virulence. Mol Microbiol 82:904-916. https://doi.org/10. 1111/j.1365-2958.2011.07862.x

Bergmann B, Raffelsbauer D, Kuhn M, Goetz M, Hom S, Goebel W (2002) InlA- but not InlB-mediated internalization of Listeria monocytogenes by non-phagocytic mammalian cells needs the support of other internalins. Mol Microbiol 43:557-570 
Burnside K, Rajagopal L (2011) Aspects of eukaryotic-like signaling in Gram-positive cocci: a focus on virulence. Future Microbiol 6:747-761. https://doi.org/10.2217/fmb.11.62

Chen B, Zhang A, Xu Z, Li R, Chen H, Jin M (2011) Large-scale identification of bacteria-host crosstalk by affinity chromatography: capturing the interactions of Streptococcus suis proteins with host cells. J Proteome Res. https://doi.org/10.1021/pr200758q

Chen L et al (2005) VFDB: a reference database for bacterial virulence factors. Nucleic Acids Res 33:D325-328. https://doi.org/10.1093/ nar/gki008

Dumesnil A et al (2018) Characterization of the zinc metalloprotease of Streptococcus suis serotype 2. Vet Res 49:109. https://doi.org/ 10.1186/s13567-018-0606-y

Fang L, Zhou J, Fan P, Yang Y, Shen H, Fang W (2017) A serine/threonine phosphatase 1 of Streptococcus suis type 2 is an important virulence factor. J Vet Sci 18:439-447. https://doi.org/10.4142/ jvs.2017.18.4.439

Feng L et al (2016) The CodY regulator is essential for virulence in Streptococcus suis serotype 2. Sci Rep 6:21241. https://doi.org/ $10.1038 /$ srep21241

Ferrando ML, Willemse N, Zaccaria E, Pannekoek Y, van der Ende A, Schultsz C (2017) Streptococcal Adhesin P (SadP) contributes to Streptococcus suis adhesion to the human intestinal epithelium. PLoS ONE 12:e0175639. https://doi.org/10.1371/journal.pone. 0175639

Fittipaldi N, Segura M, Grenier D, Gottschalk M (2012) Virulence factors involved in the pathogenesis of the infection caused by the swine pathogen and zoonotic agent Streptococcus suis. Future Microbiol 7:259-279. https://doi.org/10.2217/fmb.11.149

Hirschfeld C et al (2019) Proteomic investigation uncovers potential targets and target sites of pneumococcal serine-threonine kinase StkP and phosphatase PhpP. Front Microbiol 10:3101. https://doi. org/10.3389/fmicb.2019.03101

Ibrahim YM, Kerr AR, McCluskey J, Mitchell TJ (2004) Role of HtrA in the virulence and competence of Streptococcus pneumoniae. Infect Immun 72:3584-3591. https://doi.org/10.1128/IAI.72.6. 3584-3591.2004

Jing HB et al (2008) Proteome analysis of Streptococcus suis serotype 2. Proteomics 8:333-349. https://doi.org/10.1002/pmic.20060 0930

Ju CX, Gu HW, Lu CP (2012) Characterization and functional analysis of atl, a novel gene encoding autolysin in Streptococcus suis. J Bacteriol 194:1464-1473. https://doi.org/10.1128/JB.06231-11

Kim GL et al (2017) Effect of decreased BCAA synthesis through disruption of ilvC gene on the virulence of Streptococcus pneumoniae. Arch Pharm Res 40:921-932. https://doi.org/10.1007/ s12272-017-0931-0

Lin X et al (2015) An integrated quantitative and targeted proteomics reveals fitness mechanisms of Aeromonas hydrophila under oxytetracycline stress. J Proteome Res 14:1515-1525. https://doi. org/10.1021/pr501188g

Lun ZR, Wang QP, Chen XG, Li AX, Zhu XQ (2007) Streptococcus suis: an emerging zoonotic pathogen. Lancet Infect Dis 7:201209. https://doi.org/10.1016/S1473-3099(07)70001-4

Lyon WR, Caparon MG (2004) Role for serine protease HtrA (DegP) of Streptococcus pyogenes in the biogenesis of virulence factors SpeB and the hemolysin streptolysin S. Infect Immun 72:1618-1625

Ma F, Guo X, Fan H (2017) Extracellular nucleases of Streptococcus equi subsp. zooepidemicus degrade neutrophil extracellular traps and impair macrophage activity of the host. Appl Environ Microbiol. https://doi.org/10.1128/AEM.02468-16

Manuse S, Fleurie A, Zucchini L, Lesterlin C, Grangeasse C (2016) Role of eukaryotic-like serine/threonine kinases in bacterial cell division and morphogenesis. FEMS Microbiol Rev 40:41-56. https://doi.org/10.1093/femsre/fuv041
Moulin P et al (2016) The Adc/Lmb system mediates zinc acquisition in streptococcus agalactiae and contributes to bacterial growth and survival. J Bacteriol 198:3265-3277. https://doi.org/10.1128/ JB.00614-16

Nagy TA, Moreland SM, Andrews-Polymenis H, Detweiler CS (2013) The ferric enterobactin transporter Fep is required for persistent Salmonella enterica serovar typhimurium infection. Infect Immun 81:4063-4070. https://doi.org/10.1128/IAI.00412-13

Norris MH, Propst KL, Kang Y, Dow SW, Schweizer HP, Hoang TT (2011) The Burkholderia pseudomallei Deltaasd mutant exhibits attenuated intracellular infectivity and imparts protection against acute inhalation melioidosis in mice. Infect Immun 79:4010-4018. https://doi.org/10.1128/IAI.05044-11

Parandhaman DK, Sharma P, Bisht D, Narayanan S (2014) Proteome and phosphoproteome analysis of the serine/threonine protein kinase E mutant of Mycobacterium tuberculosis. Life Sci 109:116-126. https://doi.org/10.1016/j.lfs.2014.06.013

Pian Y et al (2015) Proteomics identification of novel fibrinogen-binding proteins of Streptococcus suis contributing to antiphagocytosis. Front Cell Infect Microbiol 5:19. https://doi.org/10.3389/ fcimb.2015.00019

Plumptre CD et al (2014) AdcA and AdcAII employ distinct zinc acquisition mechanisms and contribute additively to zinc homeostasis in Streptococcus pneumoniae. Mol Microbiol 91:834-851. https://doi.org/10.1111/mmi.12504

Ribardo DA, Hendrixson DR (2011) Analysis of the LIV system of Campylobacter jejuni reveals alternative roles for LivJ and LivK in commensalism beyond branched-chain amino acid transport. J Bacteriol 193:6233-6243. https://doi.org/10.1128/JB.05473-11

Rungelrath V et al (2018) IgM cleavage by Streptococcus suis reduces $\operatorname{IgM}$ bound to the bacterial surface and is a novel complement evasion mechanism. Virulence. https://doi.org/10.1080/21505594. 2018.1496778

Segura M, Fittipaldi N, Calzas C, Gottschalk M (2017) Critical Streptococcus suis virulence factors: are they all really critical? Trends Microbiol. https://doi.org/10.1016/j.tim.2017.02.005

Shen X et al (2013) Proteome analysis of the two-component SalK/SalR system in epidemic Streptococcus suis serotype 2. Curr Microbiol 67:118-122. https://doi.org/10.1007/s00284-013-0343-4

Si Y et al (2009) Contribution of glutamine synthetase to the virulence of Streptococcus suis serotype 2. Vet Microbiol 139:80-88. https://doi.org/10.1016/j.vetmic.2009.04.024

Tang J et al (2006) Streptococcal toxic shock syndrome caused by Streptococcus suis serotype 2. PLoS Med 3:e151. https://doi.org/ 10.1371/journal.pmed.0030151

Unwin RD, Griffiths JR, Whetton AD (2010) Simultaneous analysis of relative protein expression levels across multiple samples using iTRAQ isobaric tags with 2D nano LC-MS/MS. Nat Protoc 5:1574-1582. https://doi.org/10.1038/nprot.2010.123

Willenborg J et al (2011) Role of glucose and CcpA in capsule expression and virulence of Streptococcus suis. Microbiology 157:18231833. https://doi.org/10.1099/mic.0.046417-0

Wilson TL et al (2007) A novel signature-tagged mutagenesis system for Streptococcus suis serotype 2. Vet Microbiol 122:135-145. https://doi.org/10.1016/j.vetmic.2006.12.025

Wright DP, Ulijasz AT (2014) Regulation of transcription by eukaryotic-like serine-threonine kinases and phosphatases in Grampositive bacterial pathogens. Virulence 5:863-885. https://doi.org/ $10.4161 / 21505594.2014 .983404$

Wu Z et al (2014) The Streptococcus suis transcriptional landscape reveals adaptation mechanisms in pig blood and cerebrospinal fluid. RNA 20:882-898. https://doi.org/10.1261/rna.041822.113

Xiao G et al (2017) Streptococcus suis small RNA rss04 contributes to the induction of meningitis by regulating capsule synthesis and by inducing biofilm formation in a mouse infection model. Vet 
Microbiol 199:111-119. https://doi.org/10.1016/j.vetmic.2016. 12.034

$\mathrm{Yu} Y$ et al (2018) Infection and adaption-based proteomic changes of Streptococcus suis serotype 2 in a pig model. J Proteomics 180:41-52. https://doi.org/10.1016/j.jprot.2017.12.001

Zhang A, Mu X, Chen B, Han L, Chen H, Jin M (2011) IgA1 protease contributes to the virulence of Streptococcus suis. Vet Microbiol 148:436-439. https://doi.org/10.1016/j.vetmic.2010.09.027

Zhang $\mathrm{H}$ et al (2014) The identification of six novel proteins with fibronectin or collagen type I binding activity from Streptococcus suis serotype 2. J Microbiol 52:963-969. https://doi.org/10. 1007/s12275-014-4311-x

Zhang X et al (2015) DnaJ of streptococcus suis type 2 contributes to cell adhesion and thermotolerance. J Microbiol Biotechnol 25:771-781

Zhang C et al (2017) The eukaryote-like serine/threonine kinase STK regulates the growth and metabolism of zoonotic Streptococcus suis. Front Cell Infect Microbiol 7:66. https://doi.org/10.3389/ fcimb.2017.00066

Zheng F et al (2011) Contribution of the Rgg transcription regulator to metabolism and virulence of Streptococcus suis serotype 2. Infect Immun 79:1319-1328. https://doi.org/10.1128/IAI.00193-10

Zheng L, Khemlani A, Lorenz N, Loh JM, Langley RJ, Proft T (2015) Streptococcal 5'-nucleotidase A (S5nA), a novel streptococcus pyogenes virulence factor that facilitates immune evasion. J Biol Chem 290:31126-31137. https://doi.org/10.1074/jbc.M115. 677443

Zheng C et al (2018a) Role of two-component regulatory systems in the virulence of Streptococcus suis. Microbiol Res 214:123-128. https://doi.org/10.1016/j.micres.2018.07.002
Zheng F et al (2018b) Identification of oligopeptide-binding protein $(\mathrm{OppA})$ and its role in the virulence of Streptococcus suis serotype 2. Microb Pathog 118:322-329. https://doi.org/10.1016/j.micpa th. 2018.03 .061

Zhong X et al (2018) The two-component signaling system VraSRSS is critical for multidrug resistance and full virulence in Streptococcus suis serotype 2. Infect Immun. https://doi.org/10.1128/ IAI.00096-18

Zhu H et al (2011) The novel virulence-related gene stp of Streptococcus suis serotype 9 strain contributes to a significant reduction in mouse mortality. Microb Pathog 51:442-453. https://doi.org/10. 1016/j.micpath.2011.08.002

Zhu H et al (2014) Contribution of eukaryotic-type serine/threonine kinase to stress response and virulence of Streptococcus suis. PLoS ONE 9:e91971. https://doi.org/10.1371/journal.pone.00919 71

Zhu $\mathrm{H}$ et al (2018) The redox-sensing regulator rex contributes to the virulence and oxidative stress response of Streptococcus suis serotype 2. Front Cell Infect Microbiol 8:317. https://doi.org/10.3389/ fcimb.2018.00317

Publisher's Note Springer Nature remains neutral with regard to jurisdictional claims in published maps and institutional affiliations. 